\title{
Identifying older adults at risk of harm following elective surgery: a systematic review and meta-analysis
}

Jennifer Watt ${ }^{1,2}$, Andrea C. Tricco 3,4 , Catherine Talbot-Hamon', Ba' Pham ${ }^{3,5}$, Patricia Rios ${ }^{3}$, Agnes Grudniewicz ${ }^{6}$, Camilla Wong ${ }^{3}$, Douglas Sinclair ${ }^{3}$ and Sharon E. Straus ${ }^{1,3^{*}}$

\begin{abstract}
Background: Elective surgeries can be associated with significant harm to older adults. The present study aimed to identify the prognostic factors associated with the development of postoperative complications among older adults undergoing elective surgery.

Methods: Medline, EMBASE, CINAHL, Cochrane Central Register of Controlled Trials, and AgeLine were searched for articles published between inception and April 21, 2016. Prospective studies reporting prognostic factors associated with postoperative complications (composite outcome of medical and surgical complications), functional decline, mortality, post-hospitalization discharge destination, and prolonged hospitalization among older adults undergoing elective surgery were included. Study characteristics and prognostic factors associated with the outcomes of interest were extracted independently by two reviewers. Random effects meta-analysis models were used to derive pooled effect estimates for prognostic factors and incidences of adverse outcomes.

Results: Of the 5692 titles and abstracts that were screened for inclusion, 44 studies (12,281 patients) reported on the following adverse postoperative outcomes: postoperative complications $(n=28)$, postoperative mortality $(n=11)$, length of hospitalization $(n=21)$, functional decline $(n=6)$, and destination at discharge from hospital $(n=13)$. The pooled incidence of postoperative complications was $25.17 \%$ (95\% confidence interval (CI) 18.03-33.98\%, number needed to follow =4). The geriatric syndromes of frailty (odds ratio (OR) 2.16, 95\% Cl 1.29-3.62) and cognitive impairment (OR 2.01, 95\% Cl 1.44-2.81) were associated with developing postoperative complications; however, there was no association with traditionally assessed prognostic factors such as age (OR 1.07, 95\% Cl 1.00-1.14) or American Society of Anesthesiologists status (OR 2.62, 95\% Cl 0.78-8.79). Besides frailty, other potentially modifiable prognostic factors, including depressive symptoms (OR 1.77, 95\% Cl 1.22-2.56) and smoking (OR 2.43, 95\% Cl 1.32-4.46), were also associated with developing postoperative complications.

Conclusion: Geriatric syndromes are important prognostic factors for postoperative complications. We identified potentially modifiable prognostic factors (e.g., frailty, depressive symptoms, and smoking) associated with developing postoperative complications that can be targeted preoperatively to optimize care.
\end{abstract}

Keywords: Postoperative complications, Mortality, Functional decline, Elective surgery, Systematic review, Meta-analysis

\footnotetext{
* Correspondence: sharon.straus@utoronto.ca

'Division of Geriatric Medicine, University of Toronto, 27 King's College Circle,

Toronto, Ontario M5S 1A1, Canada

${ }^{3}$ Li Ka Shing Knowledge Institute, St. Michael's Hospital, 209 Victoria Street,

Toronto, Ontario M5B 1W8, Canada

Full list of author information is available at the end of the article
} International License (http://creativecommons.org/licenses/by/4.0/), which permits unrestricted use, distribution, and reproduction in any medium, provided you give appropriate credit to the original author(s) and the source, provide a link to the Creative Commons license, and indicate if changes were made. The Creative Commons Public Domain Dedication waiver (http://creativecommons.org/publicdomain/zero/1.0/) applies to the data made available in this article, unless otherwise stated. 


\section{Background}

As the number of older adults increases globally, there will be a greater need for elective surgeries in this patient population; however, elective surgeries can be associated with significant harm to patients [1-5]. Special preoperative consideration must be given to the greater prevalence of geriatric syndromes faced by older adults, such as frailty and functional impairment, that potentially increase their risk of adverse postoperative outcomes [6, 7]. Indeed, older adults are a heterogeneous group of patients whose risk of adverse postoperative outcomes is not adequately described by chronological age, comorbidities, or the type of surgical procedure alone [8]. Although older adults are often seen in the preoperative medicine clinic for cardiovascular and respiratory risk stratification and optimization in anticipation of an elective surgery, little consideration is given to risk stratification for other adverse outcomes that occur in older adults, despite the availability of information to aid in this assessment [9].

Understanding the risk factors for postoperative complications may help clinicians, patients, and caregivers to target non-pharmacological and pharmacological interventions aimed at lessening the burden of these adverse postoperative outcomes. This systematic review synthesizes studies that identify preoperative prognostic factors of older adults undergoing elective surgery which may predispose them to adverse postoperative outcomes. This information can be used by clinicians and patients to enhance decision-making and management in the preoperative setting and by researchers to study possible interventions aimed at improving postoperative outcomes for older adults.

\section{Methods}

This study was reported in accordance with both the PRISMA statement for reporting systematic reviews and meta-analyses and the MOOSE statement for reporting meta-analysis of observation studies in epidemiology (Additional file 1) [10, 11]. This systematic review and meta-analysis has a companion publication that focuses on prognostic factors associated with postoperative delirium among older adults undergoing elective surgery.

\section{Eligibility criteria}

Prospective studies (e.g., randomized controlled trials (RCTs), quasi-RCTs, non-RCTs, controlled-before-andafter studies, prospective cohort studies) were eligible if they included older adults undergoing elective surgery ( $\geq 60$ years old and mean age of patients enrolled in the study $\geq 65$ years old) and reported prognostic factors associated with the postoperative complications of mortality, functional decline, prolonged length of hospitalization, discharge to a location other than home, and a composite outcome of medical or surgical complications. All definitions of a given prognostic factor were included. Studies that included patients $\geq 60$ years old were selected to align with definitions from the United Nations and the World Health Organization [12, 13]. Geriatric medicine consultation services typically target these age ranges $[14,15]$. Studies using any method for diagnosing postoperative complications were eligible. Postoperative mortality was defined as death within 30 days following surgery. If a study reported both elective and emergent surgical procedures, it was included in our systematic review only if there was a separate subgroup reported for patients undergoing elective surgery. To make the review feasible, studies reporting only clinical, laboratory, or imaging investigations that are not conducted as part of routine clinical practice (i.e., measuring serum interleukin levels) were excluded, as were studies disseminated in languages other than English.

\section{Information sources and search strategy}

An experienced librarian searched MEDLINE (OVID interface, 1948 to April Week 3, 2016), EMBASE (OVID interface, 1980 to April Week 3, 2016), CINAHL (EBSCO interface, 1994 to April 21, 2016), Cochrane Central Register of Controlled Trials (Issue 4, April 2016), and AgeLine (EBSCO interface, 1968 to April 21, 2016) for potentially relevant studies. The full search strategy for MEDLINE (Additional file 2: Appendix 1) was modified as necessary for the other databases (full searches available upon request). Scanning the reference lists of included studies and searching the authors' personal files supplemented the electronic search. Authors of conference proceedings were contacted to obtain unpublished work.

\section{Study selection}

Two levels of screening were completed independently by two reviewers using Synthesi.SR (proprietary online software developed by the Knowledge Translation Program, Toronto, Canada); these were level 1 screening of titles and abstracts, and level 2, full-text screening of articles. A calibration exercise was conducted prior to level 1 screening whereby each reviewer independently screened $10 \%$ of a random sample of citations to ensure adequate interrater agreement. Study authors were contacted for further information if it was unclear whether the study met inclusion criteria. Disagreements concerning article inclusion were resolved through discussion; otherwise, a third reviewer was available to make a final decision.

\section{Data abstraction}

Data were abstracted independently by two reviewers from studies retained from level 2 screening. Study characteristics (e.g., study design, country of conduct), patient characteristics (e.g., mean age, sex, comorbidities), and prognostic factors associated with the outcomes of interest were abstracted from included studies. Definitions operationalized 
by study authors for individual prognostic factors were also abstracted, where appropriate. Conflicts regarding the abstracted data were resolved through discussion. Authors were contacted for further information when the data were not clearly reported. The publication with the longest duration of follow-up was considered the major publication when multiple studies reported data from the same source. The other publications were retained as supplementary material only.

\section{Methodological quality assessment}

Two reviewers independently appraised the risk of bias using the Cochrane Risk-of-Bias Tool for RCTs and the Newcastle-Ottawa Scale for cohort studies $[16,17]$. We planned to assess other study designs with the Cochrane Effective Practice and Organization Care (EPOC) Risk-ofBias Tool [18]. If two or more outcomes were reported in a single study, the quality assessment was preferentially conducted on the outcome of postoperative complications or destination at discharge from hospital.

\section{Statistical methods}

We calculated odds ratios (OR) to quantify the relative risk of postoperative complications associated with each prognostic factor. Whenever only continuous effect measures, such as mean differences (e.g., age, body mass index) were reported, these effect sizes were transformed to OR estimates, if needed, to derive an overall effect estimate that combined both dichotomous and continuous study-level effect estimates [19]. For studies that reported multiple options with which to derive the study-level effect estimate (e.g., $2 \times 2$ tables, adjusted and unadjusted ORs, mean differences), the order of preference for selecting the source data is described in Additional file 2: Appendix 2.

Random effects models were used to derive overall effect estimates with $95 \%$ confidence intervals (CIs) when two or more studies reported extractable effect estimates that could be combined for the purpose of metaanalysis. The number needed to follow (NNF) was calculated as 1 /pooled incidence of each postoperative complication. Similar to the concept of the number needed to treat or number needed to harm, the NNF represents the number of patients who need to be followed in a prognostic study in order to see one outcome [20]. Information regarding data imputation methods to approximate standard deviation values is found in Additional file 2: Appendix 3. Between-study statistical heterogeneity was quantitatively assessed with the $\mathrm{I}^{2}$ statistic and thresholds for the interpretation of the $\mathrm{I}^{2}$ statistic were consistent with those reported in the Cochrane Handbook for Systematic Reviews of Interventions [21].

Subgroup analyses were conducted by surgery type to explore between-study heterogeneity. Mixed-effects meta- regression models were also used to evaluate the effect of study-level effect modifiers (age, publication year, and type of surgery) on the pooled incidence of postoperative complications. Sensitivity analyses were conducted based on the type of study-level effect estimates used to calculate the overall effect estimates, - including study-level effect estimates that were adjusted for potentially important confounders only. A prognostic factor was considered significantly associated with the primary or secondary outcomes at a two-tailed $p$-value $<0.05$. We planned to test for publication bias; however, this was not possible because there were no prognostic factors that were reported in at least 10 studies. All statistical analyses were conducted in R, version 3.2.4, using the metafor and meta packages [22, 23].

\section{Results}

Of the 5692 titles and abstracts that were screened for inclusion, 44 studies, including 12,281 patients, met our inclusion criteria (Fig. 1). From the 44 included studies, prognostic factors associated with postoperative complications $(n=28)$, postoperative mortality $(n=10)$, length of hospitalization $(n=22)$, functional decline $(n=6)$, and destination at discharge from hospital $(n=13)$ were retrieved. Two RCTs were included, which were at moderate to high risk of bias (Additional file 2: Appendix 4). Overall, the included cohort studies were of moderate to high methodological quality (Additional file 2: Appendix 5). The most common biases were the adequacy of follow-up of cohorts and the comparability of the cohorts on the basis of design.

\section{Postoperative complications}

Twenty-eight studies (6708 patients) investigated the association between preoperative prognostic factors and postoperative complications (Additional file 1: Appendix 6). Of these, 23 were included in the meta-analyses of prognostic factors $[1,6,7,24-43]$. The five studies not included in meta-analyses did not contain extractable data, report prognostic factors that were included in two or more studies, or present data in a format that could be pooled with other study-level effect estimates. Postoperative complications were most often reported as a composite of postoperative medical or surgical complications (e.g., pneumonia, wound infection, venous thromboembolism), the details of which are found in Additional file 2: Appendix 6. The pooled incidence of postoperative complications across all surgical types was $25.16 \%$ (95\% CI 18.26-33.61\%, 21 studies, $\left.\mathrm{I}^{2}=96 \%, \mathrm{NNF}=4\right)[1,7,25-28,30-33,35-38,40-46]$. In exploring the influence of type of surgery on incident complications, the number of complications remained high: cardiac surgery $(9.46 \%, 95 \%$ CI $2.71-28.18 \%, 3$ studies, $\left.\mathrm{I}^{2}=96.40 \%, \mathrm{NNF}=11\right)$, abdominal surgery $(24.73 \%$, 
from the electronic database search

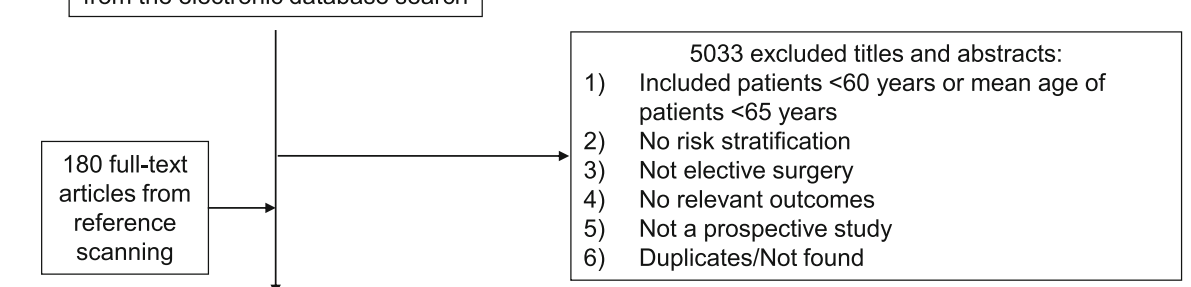

765 excluded full-text studies:

1) No risk stratification (40)

2) Not elective surgery (50)

3) Included patients $<60$ years or mean age of patients $<65$ years $(360)$

4) Not written in English (18)

5) Not a prospective study (235)

6) Unable to obtain data (30)

7) No relevant outcomes (55)

8) Not a test done in routine pre-op assessment (2)

44 studies included in the qualitative synthesis Systematic reviews (5)

26 studies included in the quantitative synthesis of risk factors for postoperative harm

Fig. 1 Study flow

95\% CI 8.63-53.33\%, 3 studies, $\mathrm{I}^{2}=96.1 \%, \mathrm{NNF}=5$ ), and thoracic surgery $(33.97 \%, 95 \%$ CI $12.66-64.62 \%$, 4 studies, $\mathrm{I}^{2}=95.5 \%, \mathrm{NNF}=3$ ). The effects of the mean age of study patients, publication year, and type of surgery on the pooled incidence of postoperative complications were explored with meta-regression, but did not explain any of the variance in the models.

The prognostic factors most strongly associated with the development of postoperative complications were poor performance status as defined by the Eastern Cooperative Oncology Group (ECOG) score or the Karnofsky Index (OR 2.58, 95\% CI 1.56-4.25, 5 studies, $\mathrm{I}^{2}=0 \%$ ), smoking status (OR 2.43, 95\% CI 1.32-4.46, 3 studies, $\mathrm{I}^{2}=0 \%$ ), impairment in instrumental activities of daily living (IADLs) (OR 2.27, 95\% CI 1.65-3.14, 6 studies, $\mathrm{I}^{2}=0 \%$ ), frailty (OR 2.16 , 95\% CI $1.29-3.62,8$ studies, $\mathrm{I}^{2}=54.69 \%$ ), and cognitive impairment (OR 2.01, 95\% CI 1.44-2.81, 8 studies, $\mathrm{I}^{2}=0 \%$ ) (Table 1, Additional file 2: Appendix 7). Frailty was most frequently defined using the definition of Fried et al. [47]; however, other definitions included the Edmonton Frailty Scale, gait speed, or a tool created by individual study authors [48]. In a subgroup of frail patients undergoing abdominal surgery, there was no longer an association between frailty and postoperative complications (OR 1.73, 95\% CI $0.81-3.66,3$ studies, $\left.\mathrm{I}^{2}=53.36 \%\right) \quad[29,35,40]$. These findings remained consistent when sensitivity analyses were conducted whereby only those studies reporting study-level effect estimates adjusted for important confounders were included.
Other prognostic factors that were reported in single studies as significantly associated with postoperative complications were the cumulative number of impairments in the comprehensive geriatric assessment (OR 1.84, 95\% CI 1.27-2.65), not being able to shop independently $(P=0.011)$, answering 'yes' to the question 'Have you dropped many of your activities and interests?' on the Geriatric Depression Scale (GDS) [49] $(P=0.04)$, the presence of one or more Goldman indicators [50] $(P<0.005)$, and the inability to bicycle 2 minutes to a heart rate greater than 99 beats/min $(P<0.05)[44,45,51]$. The presence of anxiety (OR 5.1, 95\% CI 1.27-20.2), Society of Thoracic Surgeons score [52] (OR 1.06, 95\% CI 1.01-1.10), and female sex (OR 3.49, 95\% CI 1.52-7.99) were associated with mortality or major morbidity in patients undergoing cardiac surgery [53].

Energy intake $>21.3 \mathrm{kcal} / \mathrm{kg}$ of actual body weight (OR 2.40, 95\% CI 0.59-9.80), energy intake > $22.2 \mathrm{kcal} / \mathrm{kg}$ of ideal body weight (OR 5.00, 95\% CI 0.95-26.17), or any of the items on the Nutrition Screening Initiative Nutritional Health Checklist [54] were not associated with postoperative complications $[45,46]$. Besides answering 'yes' to the question 'Have you dropped many of your activities and interests?' on the GDS, none of the other questions were associated with postoperative complications. Similarly, no activities of daily living (ADLs) or IADLs, besides shopping, were individually associated with postoperative complications [45]. 
Table 1 Prognostic factors for postoperative complications among older adults undergoing elective surgery

\begin{tabular}{|c|c|c|c|c|}
\hline Prognostic factor & Number of studies & Number of patients & Odds ratio (95\% Cl) & Heterogeneity $\left(1^{2}\right)$ \\
\hline Poor performance status & 5 & 889 & $2.58(1.56-4.25)$ & 0 \\
\hline Smoking status & 3 & 907 & $2.43(1.32-4.46)$ & 0 \\
\hline IADL impairment & 7 & 1036 & $2.27(1.65-3.14)$ & 0 \\
\hline Frailty & 8 & 1527 & $2.16(1.29-3.62)$ & 54.69 \\
\hline Cognitive impairment & 8 & 1851 & $2.01(1.44-2.81)$ & 0 \\
\hline ADL impairment & 4 & 829 & $1.98(1.31-2.99)$ & 0 \\
\hline Geriatric depression screen & 4 & 777 & $1.77(1.22-2.56)$ & 0 \\
\hline Comorbidity score & 5 & 1000 & $1.55(1.29-1.87)$ & 0 \\
\hline Depression & 2 & 257 & $2.04(0.67-6.23)$ & 0 \\
\hline Poor mobility & 2 & 477 & $2.51(0.92-6.84)$ & 63.37 \\
\hline Older age & 9 & 2917 & $1.07(1.00-1.14)$ & 17.96 \\
\hline General anesthesia & 2 & 172 & $0.78(0.38-1.59)$ & 0 \\
\hline ASA score $\geq 3$ & 3 & 420 & $2.62(0.78-8.79)$ & 0 \\
\hline Malnutrition & 7 & 847 & $1.22(0.66-2.24)$ & 31.02 \\
\hline Hypertension & 3 & 912 & $0.90(0.52-1.54)$ & 0 \\
\hline Cerebrovascular disease & 2 & 845 & $0.81(0.11-5.94)$ & 83.39 \\
\hline Diabetes mellitus & 3 & 912 & $0.70(0.39-1.26)$ & 0 \\
\hline Polypharmacy & 4 & 442 & $1.46(0.9-2.37)$ & 0 \\
\hline Male sex & 6 & 2141 & $1.60(0.88-2.91)$ & 66.24 \\
\hline
\end{tabular}

$A D L$ activities of daily living, $A S A$ American Society of Anesthesiologists, $I A D L$ instrumental activities of daily living, $C l$ confidence interval

\section{Postoperative mortality}

The association between preoperative prognostic factors and postoperative mortality was investigated in 11 studies (3399 patients) (Table 2) [1-3, 25, 30, 32, 51, 53, 5557]. The pooled incidence of mortality was $4.58 \%(95 \%$ CI $3.67-5.71 \%, 11$ studies, $\left.\mathrm{I}^{2}=46.30 \%, \mathrm{NNF}=21\right)[1-3$, 25, 30, 32, 51, 53, 55-57]. Among patients undergoing cardiac surgery, the pooled incidence of mortality was $5.21 \%\left(4.00-6.75 \%, 6\right.$ studies, $\left.\mathrm{I}^{2}=60.8 \%, \quad \mathrm{NNF}=20\right)$. Only the effects of publication year could be explored in a meta-regression because there were not enough studies to explore the effects of type of surgery or mean age of patients on the pooled incidence of mortality. Publication year did not explain any of the variance in the meta-regression model. Few prognostic factors were reported in more than one study. No significant association was identified between male sex (OR 1.46, 95\% CI 0.67-3.19, 4 studies, $\mathrm{I}^{2}=53.92 \%$ ), diabetes mellitus (HR $1.74,95 \%$ CI $0.54-5.61,2$ studies, $\mathrm{I}^{2}=45.26 \%$ ), or history of heart failure (HR 1.86, 95\% CI 0.44-7.88, 2 studies, $\mathrm{I}^{2}$ $=68.34 \%$ ) and postoperative mortality (Additional file 2: Appendix 8) [1, 30, 32, 55, 56].

\section{Length of hospitalization}

A total of 21 studies (5037 patients) investigated preoperative prognostic factors and length of hospitalization (Table 3) $[1,2,7,24,25,27,29-31,35-37,39,45,46,51$,
53, 58-61]. Substantial between-study heterogeneity in the reporting of outcomes and few prognostic factors being reported in more than one study largely precluded pooling of study-level effect estimates. There was no association between higher American Society of Anesthesiologists (ASA) score and prolonged hospitalization (OR 0.82, 95\% CI 0.30-2.23, 2 studies, $\mathrm{I}^{2}=0 \%$ ) (Additional file 2: Appendix 9) [2, 31]. Among six studies that investigated frailty as a prognostic factor for prolonged hospitalization, there was a significant association identified in four studies [7, 29, 35, 37, 40, 61]. One study found that, while frail patients with postoperative complications had prolonged hospitalizations $(P<0.001)$, those without postoperative complications did not $(P=0.19)$ [36]. Age was identified as a significant prognostic factor for prolonged hospitalization in only two of six studies $[1,2,6,26,33,51,62-64]$.

\section{Destination at discharge from hospital}

In total, 13 studies (2601 patients) investigated associations between preoperative prognostic factors and the destination at discharge from hospital (e.g., skilled nursing facility vs. discharge to home) (Table 4) $[2,7,25$, 29, 37, 38, 40, 45, 51, 53, 61, 65, 66]. Patients were discharged to a number of locations, including other hospitals, nursing homes, rehabilitation centres, transitional care facilities, and assisted-living facilities. The 
Table 2 Prospective studies of risk factors for postoperative mortality among older adults undergoing elective surgery

\begin{tabular}{|c|c|c|c|c|c|}
\hline \multirow[t]{2}{*}{ Study } & \multirow{2}{*}{$\begin{array}{l}\text { Number } \\
\text { of patients }\end{array}$} & \multicolumn{2}{|c|}{ Number of deaths } & \multirow{2}{*}{$\begin{array}{l}\text { Factors associated with } \\
\text { postoperative mortality }\end{array}$} & \multirow{2}{*}{$\begin{array}{l}\text { Factors not associated with } \\
\text { postoperative mortality }\end{array}$} \\
\hline & & $N$ & $\%$ & & \\
\hline Audisio, 2008 [1] & 460 & 16 & 3.5 & $\begin{array}{l}\text { Male sex ( } 6.5 \% \text { vs. } 2.0 \%) \text {, more } \\
\text { advanced cancer stage }(P=0.001)\end{array}$ & Age $(P>0.05)$ \\
\hline Badgwell, 2013 [2] & 111 & 2 & 2 & NR & $\begin{array}{l}\text { No clinical, demographic, or CGA } \\
\text { results were associated with morbidity or death }\end{array}$ \\
\hline Betomvuko, 2015 [57] & 94 & 4 & 4.2 & $\begin{array}{l}\text { Gait speed }(0.68 \pm 0.23 \mathrm{~m} / \mathrm{s} \text { vs. } \\
0.43 \pm 0.06 \mathrm{~m} / \mathrm{s}, P=0.037)\end{array}$ & NR \\
\hline Gerude, 2014 [30] & 67 & 3 & 4.5 & Male sex, IADL impairment & NR \\
\hline Javierre, 2012 [32] & 2038 & 74 & 3.6 & Age (OR 2.28, 95\% Cl 1.52-3.43) & Male sex (OR 0.93, 95\% Cl 0.56-1.56) \\
\hline Kim, 2013 [51] & 141 & 6 & 4.3 & NR & $\begin{array}{l}\text { Cumulative number of impairments } \\
\text { on CGA (OR } 1.216,95 \% \mathrm{Cl} 0.864-1.712 \text {, } \\
\text { for death or post-discharge } \\
\text { institutionalization) }\end{array}$ \\
\hline Sundermann, 2014 [3] & 455 & 28 & 6.1 & $\begin{array}{l}\text { CAF score (OR 1.1, 95\% Cl } \\
1.06-1.12), \text { FORECAST score } \\
\text { (OR 1.3, 95\% Cl 1.2-1.5), } \\
\text { EuroSCORE (OR 1.1, 95\% Cl } \\
1.03-1.1), \text { STS score (OR } 1.3 \\
(95 \% \text { Cl 1.1-1.5) }\end{array}$ & NR \\
\hline Tamburino, 2011 [55] & 663 & 39 & 5.9 & $\begin{array}{l}\text { Diabetes mellitus (HR 2.66, } \\
95 \% \mathrm{Cl} 1.26-5.65), \text { LVEF } \\
<40 \% \text { (HR 3.51, 95\% Cl } \\
1.62-7.62)\end{array}$ & EuroSCORE (c-statistic 0.55) \\
\hline Wenaweser, 2011 [56] & 200 & 15 & 7.5 & $\begin{array}{l}\mathrm{BMI}<20(\mathrm{HR} 6.60,95 \% \mathrm{Cl} \\
1.48-29.5) \text {, stroke (HR 4.41, } \\
95 \% \mathrm{Cl} 1.16-16.8)\end{array}$ & $\begin{array}{l}\text { Age }>85 \text { years (HR 1.69, 95\% Cl 0.17-16.5), } \\
\text { male sex (HR 0.90, 95\% Cl 0.32-2.52), } \\
\text { diabetes (HR 0.75, 95\% Cl 0.21-2.67), CHF } \\
\text { (HR 0.79, 95\% Cl 0.25-2.47), COPD (HR 1.35, } \\
95 \% \mathrm{Cl} 0.38-4.79), \text { hypertension (HR 1.20, } \\
95 \% \mathrm{Cl} 0.34-4.24), \text { prior MI (HR 0.62, 95\% } \\
\mathrm{Cl} 0.14-2.75), \text { LVEF > 50\% (HR 4.42, 95\% } \\
\mathrm{Cl} 0.55-35.5) \text {, atrial fibrillation (HR 1.75, 95\% } \\
\mathrm{Cl} 0.59-5.21 \text { ) }\end{array}$ \\
\hline Williams, 2013 [53] & 148 & 7 & 4.7 & NR & Anxiety (OR 2.53, 95\% Cl 0.26-24.82) \\
\hline
\end{tabular}

BMI body mass index, CAF comprehensive assessment of frailty, CGA comprehensive geriatric assessment, CHF congestive heart failure, COPD chronic obstructive pulmonary disease, EuroSCORE European System for Cardiac Operative Risk Evaluation, FORECAST Frailty predicts death one year after cardiac surgery test, HR hazard ratio, IADL instrumental activities of daily living, LVEF left ventricular ejection fraction, MI myocardial infarction, STS Society of Thoracic Surgeons, CI confidence interval, $N$ number of patients, $N R$ not reported, $O R$ odds ratio

pooled incidence of discharge from hospital to a destination other than home was $13.65 \%(8.90-20.39 \%, 9$ studies, $\left.\mathrm{I}^{2}=91.6 \%, \mathrm{NNF}=8\right)[2,25,37,38,40,45,51,53$, 66]. In a subgroup of older adults undergoing general surgery, the pooled incidence of being discharged to a non-home location was $9.97 \%$ (6.59-12.39\%, 2 studies, $\left.\mathrm{I}^{2}=0 \%, \mathrm{NNF}=11\right)[2,40]$. Only the effects of publication year could be explored in a meta-regression because there were not enough studies to explore the effects of type of surgery or mean age of patients on the pooled incidence of mortality. Publication year did not explain any of the variance in the meta-regression model. In another subgroup of older adults, the pooled incidence of being discharged to a nursing home was $9.97 \%$ (5.30$17.96 \%, 2$ studies, $\left.\mathrm{I}^{2}=86 \%\right)[37,66]$. Meta-analysis of data from five studies (1228 patients) found that frailty was associated with non-home discharge following elective surgery (OR 3.42, 95\% CI 1.35-8.68, $\mathrm{I}^{2}=67.46 \%$ )
(Additional file 2: Appendix 10) [7, 29, 37, 38, 40]. In an additional study, the odds of being transferred to another hospital were six times greater for frail patients $(P$ $=0.002$ ) (Table 4) [61]. There were a number of prognostic factors that were associated with an increased risk of non-home destination at discharge from hospital, namely older age, weight loss $\geq 10 \%$, ASA score $\geq 2$, ECOG performance status $\geq 2$, and lower self-reported mobility $[2,7,37,66,67]$.

\section{Functional decline}

Six studies (1426 patients) investigated the association between preoperative prognostic factors and postoperative functional decline (Table 5) [63-65, 68-70]. All six studies reported prognostic factors associated with postoperative impairment in a patient's ability to perform ADLs. One study reported risk factors associated with postoperative impairment in the ability to perform IADLs [63]. The 


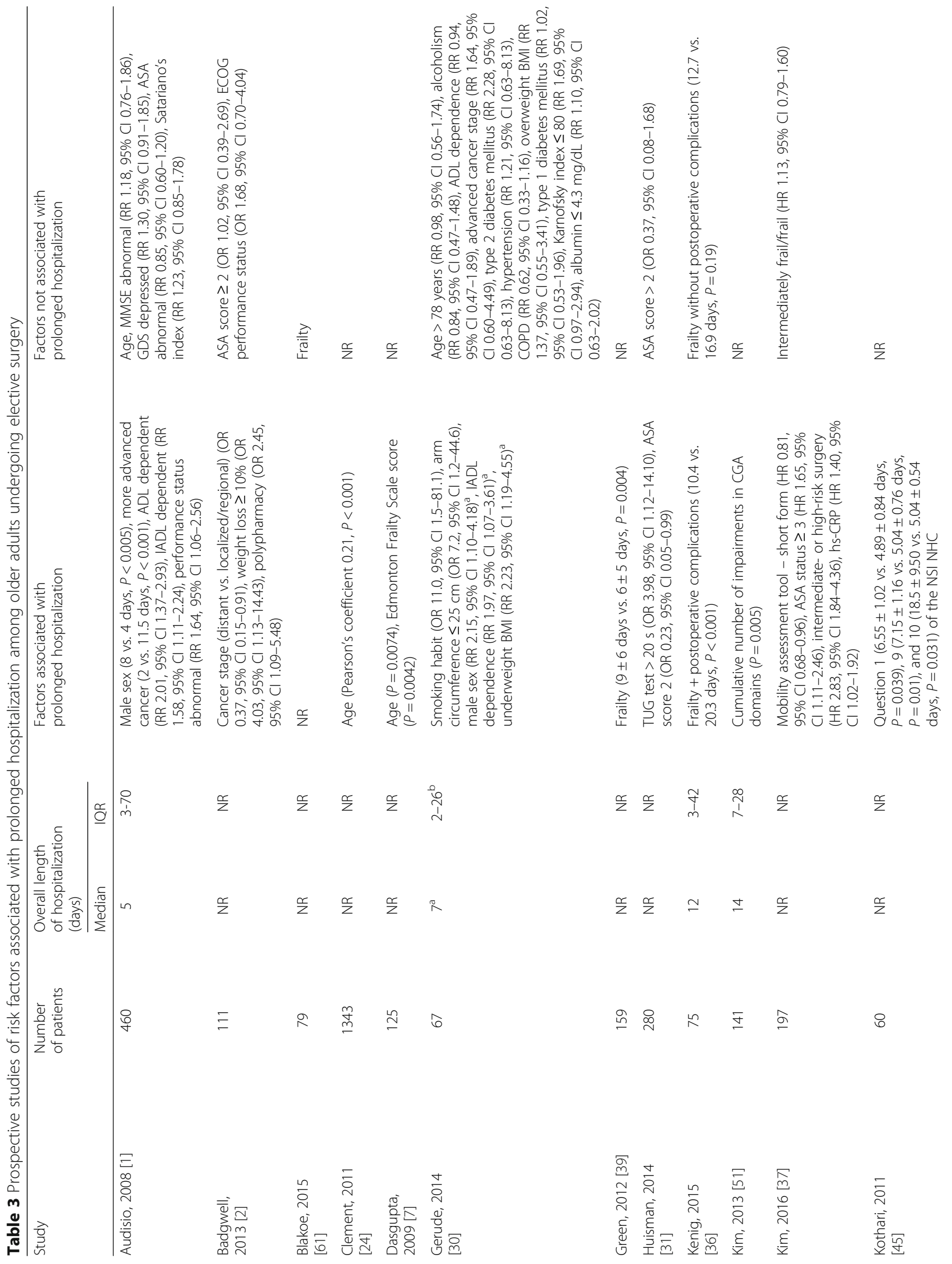




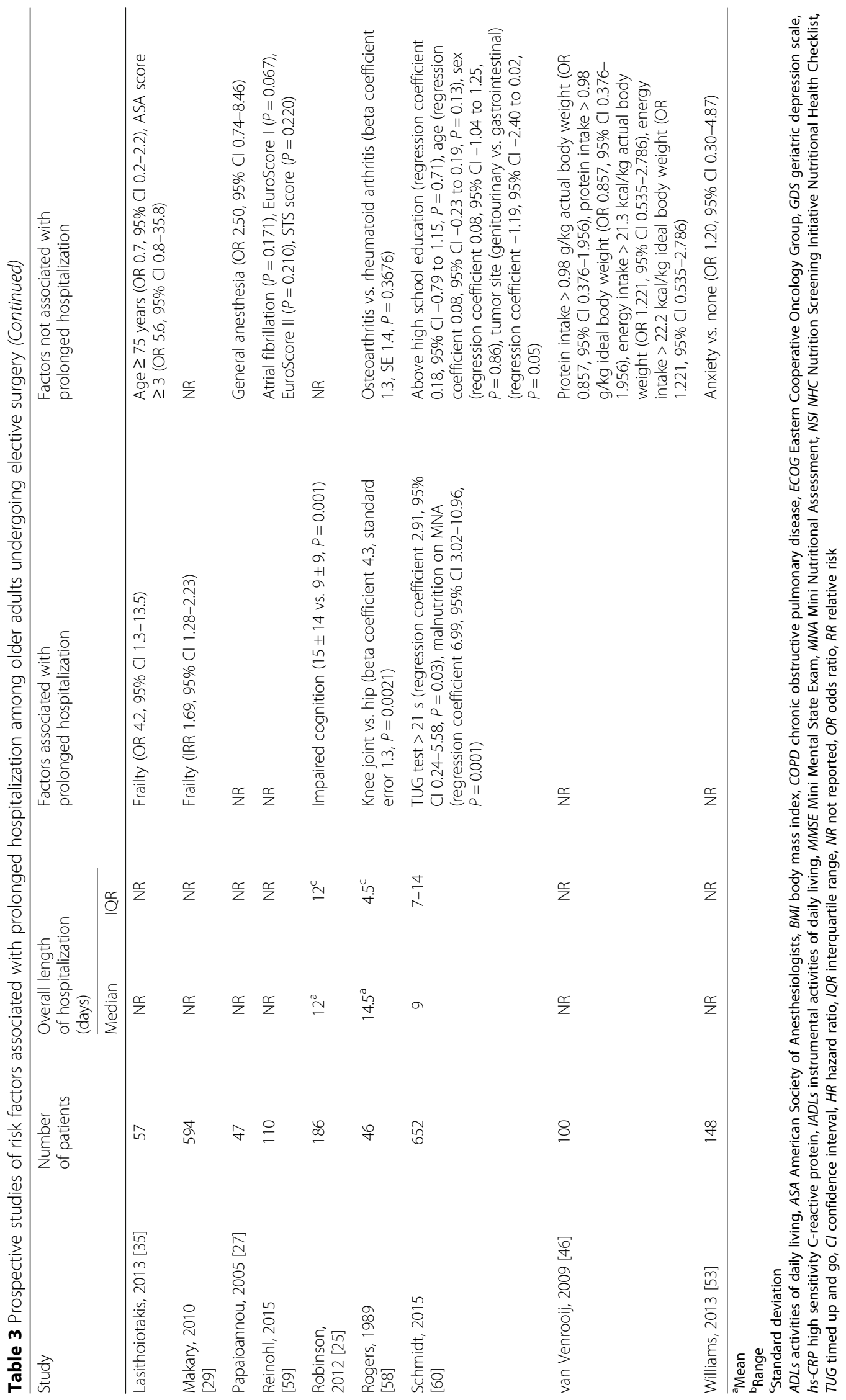


Table 4 Prospective studies of risk factors associated with non-home discharge among older adults undergoing elective surgery

\begin{tabular}{|c|c|c|c|c|c|c|}
\hline \multirow[t]{2}{*}{ Study } & \multirow[t]{2}{*}{$\begin{array}{l}\text { Number } \\
\text { of patients }\end{array}$} & \multicolumn{2}{|c|}{$\begin{array}{l}\text { Non-home } \\
\text { discharge }\end{array}$} & \multirow[t]{2}{*}{ Discharge destination } & \multirow[t]{2}{*}{$\begin{array}{l}\text { Factors associated with } \\
\text { non-home discharge }\end{array}$} & \multirow[t]{2}{*}{$\begin{array}{l}\text { Factors not associated with } \\
\text { non-home discharge }\end{array}$} \\
\hline & & $\mathrm{N}$ & $\%$ & & & \\
\hline $\begin{array}{l}\text { Badgwell, } \\
2013 \text { [2] }\end{array}$ & 111 & 11 & 10 & $\begin{array}{l}\text { Skilled nursing facility } \\
\text { (including inpatient } \\
\text { rehabilitation facilities) }\end{array}$ & $\begin{array}{l}\text { Weight loss } \geq 10 \% \text { (OR } 6.52,95 \% \\
\text { Cl 1.43-29.76), ASA score } \geq 2 \\
\text { (OR 5.08, 95\% CI 1.13-22.78), ECOG } \\
\text { performance status } \geq 2 \text { (OR 4.51, 95\% } \\
\text { Cl 1.03-19.71) }\end{array}$ & $\begin{array}{l}\text { Polypharmacy (OR 1.33, 95\% } \\
\text { Cl 0.38-4.64), distant stage } \\
\text { cancer (OR 0.54, 95\% Cl } \\
0.11-2.64)\end{array}$ \\
\hline $\begin{array}{l}\text { Blakoe, } \\
2015[61]\end{array}$ & 79 & $N R$ & NR & Another hospital & Frailty $(\mathrm{OR} 6, P=0.002)$ & NR \\
\hline $\begin{array}{l}\text { Courtney- } \\
\text { Brooks, } \\
2012 \text { [38] }\end{array}$ & 37 & 1 & 2.7 & Skilled nursing facility & $N R$ & Frailty $(P=0.25)$ \\
\hline $\begin{array}{l}\text { Dasgupta, } \\
2009[7]\end{array}$ & 125 & NR & NR & Institution & $\begin{array}{l}\text { Age }(P=0.0009) \text {, Edmonton } \\
\text { Frailty Scale score }(P=0.013)\end{array}$ & NR \\
\hline $\begin{array}{l}\text { Kim, } 2013 \\
{[51]}\end{array}$ & 141 & 26 & 19.3 & $\begin{array}{l}\text { Nursing home, transitional } \\
\text { care facility, or acute care } \\
\text { facility }\end{array}$ & NR & $\begin{array}{l}\text { Cumulative number of impairments } \\
\text { on CGA (OR } 1.216,95 \% \mathrm{Cl} 0.864- \\
1.712, \text { for death or post-discharge } \\
\text { institutionalization) }\end{array}$ \\
\hline $\begin{array}{l}\text { Kim, } 2014 \\
{[40]}\end{array}$ & 275 & 24 & 8.7 & $\begin{array}{l}\text { Nursing home, transitional } \\
\text { care, or any long-term care } \\
\text { facility }\end{array}$ & Frailty (OR 1.42, 95\% Cl 1.09-1.86) & NR \\
\hline $\begin{array}{l}\text { Kim, } 2016 \\
{[37]}\end{array}$ & 197 & 27 & 13.7 & Nursing home & $\begin{array}{l}\text { Mobility assessment tool - short form } \\
\text { (OR 2.01, 95\% CI 1.13-3.56), intermediately } \\
\text { frail/frail (OR 3.11,95\% Cl 1.02-9.54), age } \\
\text { (OR 1.15, 95\% Cl 1.05-1.27), preoperative } \\
\text { pain score (OR 0.83, 95\% Cl 0.70-0.99) }\end{array}$ & NR \\
\hline $\begin{array}{l}\text { Kothari, } \\
2011[45]\end{array}$ & 60 & 6 & 10 & $\begin{array}{l}\text { Location other than } \\
\text { home }\end{array}$ & IADL score for 'shopping' $(P=0.003)$ & $\begin{array}{l}\text { IADL scores (food preparation, } \\
\text { housekeeping, laundry, medications, } \\
\text { managing money, telephone usage, } \\
\text { transportation), NSI Nutritional Health } \\
\text { Checklist, GDS }\end{array}$ \\
\hline $\begin{array}{l}\text { Legner, } \\
2004[66]\end{array}$ & 586 & 43 & 14 & Nursing home & $\begin{array}{l}\text { Age } 70-74 \text { years vs. < } 65 \text { years (OR } 5.4 \\
95 \% \text { Cl 1.9-15.7), } 75-79 \text { years (OR 10.5, } \\
95 \% \text { Cl 3.7-29.5), } \geq 80 \text { years (OR 16.3, } \\
95 \% \text { Cl 5.5-48.7) }\end{array}$ & $\begin{array}{l}\text { Age } 65-69 \text { years vs. }<65 \\
\text { years (OR 2.5, 95\% Cl 0.8-8.3) }\end{array}$ \\
\hline $\begin{array}{l}\text { Makary, } \\
2010[29]\end{array}$ & 594 & NR & NR & $\begin{array}{l}\text { Skilled or assisted-living } \\
\text { facility }\end{array}$ & Frailty (OR 20.48, 95\% Cl 5.54-75.68) & NR \\
\hline $\begin{array}{l}\text { Min, } 2015 \\
{[65]}\end{array}$ & 49 & NR & NR & All non-home locations & NR & $\begin{array}{l}\text { Any of the baseline geriatric } \\
\text { assessments }\end{array}$ \\
\hline $\begin{array}{l}\text { Robinson, } \\
2012[25]\end{array}$ & 186 & 52 & 29 & $\begin{array}{l}\text { Institutional care facility } \\
\text { (i.e., nursing home, skilled } \\
\text { nursing facility or } \\
\text { rehabilitation centre) }\end{array}$ & $\begin{array}{l}\text { Impaired cognition (OR 3.01, 95\% } \\
\text { Cl 1.55-5.86) }\end{array}$ & NR \\
\hline $\begin{array}{l}\text { Williams, } \\
2013 \text { [53] }\end{array}$ & 148 & 47 & 31.8 & Healthcare facility & $N R$ & $\begin{array}{l}\text { Anxiety vs. none (OR 2.29, 95\% } \\
\mathrm{Cl} 0.65-8.10)\end{array}$ \\
\hline
\end{tabular}

ASA American Society of Anesthesiologists, CGA comprehensive geriatric assessment, ECOG Eastern Cooperative Oncology Group, GDS geriatric depression scale, $I A D L$ instrumental activities of daily living, NSI Nutrition Screening Initiative, $C I$ confidence interval, $N R$ not reported, $O R$ odds ratio

pooled incidence of decline in ADLs was 21.03\% (9.94$39.11 \%$, 4 studies, $\left.\mathrm{I}^{2}=97.1 \%, \mathrm{NNF}=5\right)[63,64,69,70]$. In a subgroup of patients undergoing general surgery, the pooled incidence of decline in ADLs was $15.25 \%$ $\left(5.48-35.83 \%, 2\right.$ studies, $\left.\mathrm{I}^{2}=95.7 \%, \mathrm{NNF}=7\right)[63,64]$. Age was not found to be associated with postoperative impairment in ADLs at 4-6 weeks, 3 months, or 1 year after elective surgery; however, one study did show an association between age and impairment in
ADLs in the postoperative period $[63-65,68,70]$. Baseline MMSE score was not associated with a decline in ADLs, but it was associated with a decline in IADLs [63, 64].

\section{Discussion}

This systematic review and meta-analysis identified preoperative prognostic factors associated with the risk of harm in older adults undergoing elective surgery. 


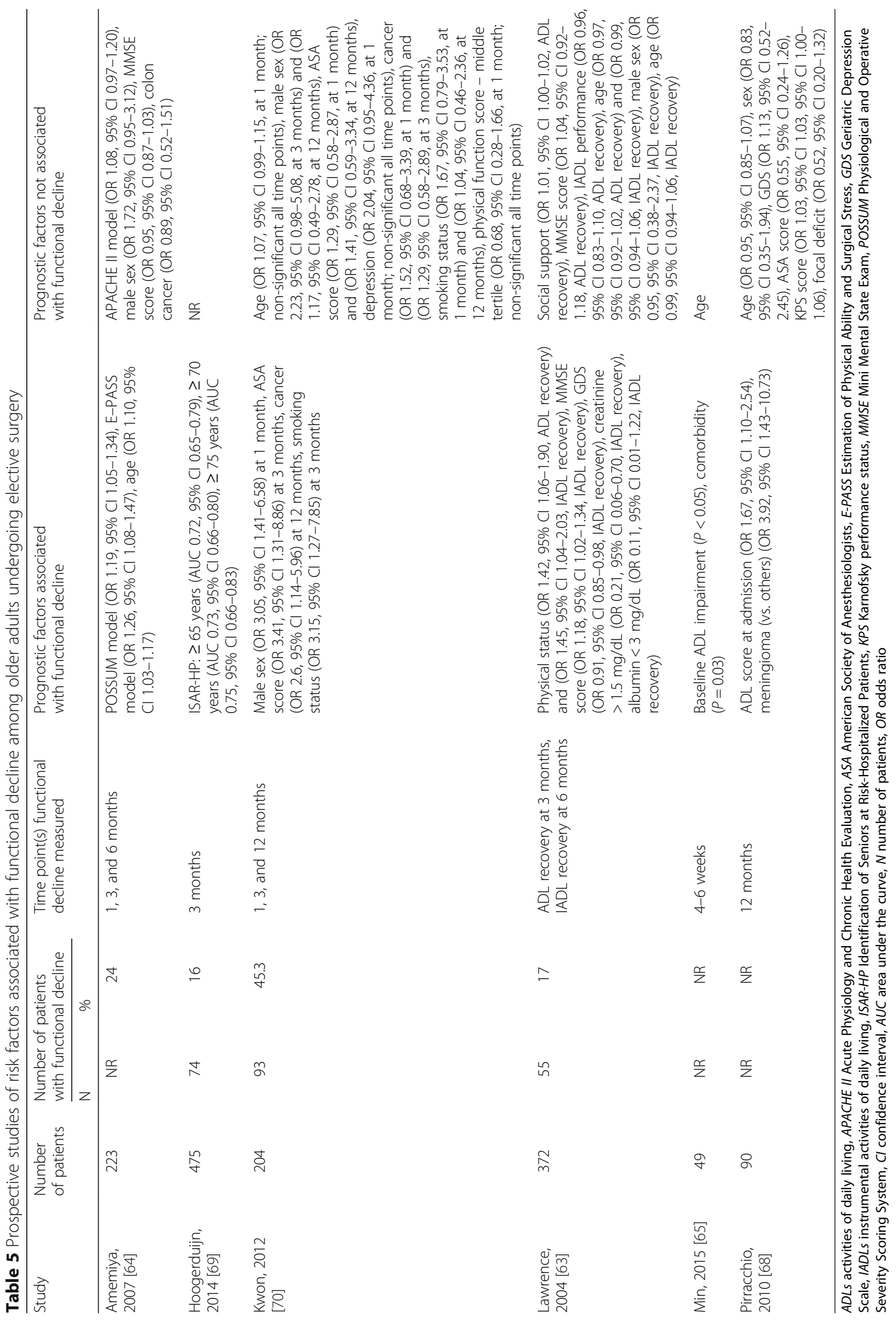


Common geriatric syndromes, such as functional impairment, cognitive impairment, and frailty, were associated with the composite outcome of postoperative complications, while more traditional perioperative risk factors in the medical literature, such as older age and ASA status, were not [71]. Although the pooled incidences of adverse postoperative outcomes must be interpreted with caution because of significant between-study heterogeneity, it is worth noting that approximately one in four older adults suffered a postoperative complication from undergoing elective surgery. Fortunately, we identified a number of potentially modifiable risk factors, including smoking status, depressive symptoms, and frailty, that can be explored in future studies aimed at preventing adverse postoperative outcomes in older adults undergoing elective surgery.

The finding that geriatric syndromes, but not older age or ASA status, were associated with postoperative complications warrants further discussion. In particular, frailty is felt to represent a patient's biological age as opposed to their chronological age, which may explain why frailty and not older age was associated with postoperative complications in this setting [72]. Frail patients were also less likely to be discharged to their home, which again likely reflects their decreased physiological reserve to respond to a significant stressor such as surgery. Besides being associated with postoperative complications, frailty has been associated with a number of other adverse outcomes outside of the perioperative literature, including mortality and admission to a long-term care facility [73, 74]. Perhaps greater emphasis should be placed on a patient's frailty status as opposed to their age in determining risk of adverse postoperative outcomes as part of a comprehensive preoperative assessment [9].

The high incidence of adverse outcomes $(25 \%$ of patients experiencing a postoperative complication), even in this non-emergent surgical setting, was also surprising. There was significant between-study heterogeneity among studies reporting postoperative complications, which could not be completely explained by type of surgery, but instead likely reflects the range of postoperative complications that were reported by study authors (e.g., atelectasis, venous thromboembolism, death). In the future, it will be important for more researchers to identify postoperative complications by severity so that knowledge users (e.g., patients, clinicians) can have a better-informed discussion as to a patient's risk of developing different postoperative complications.

To our knowledge, this is the first systematic review and meta-analysis that comprehensively examined the association between preoperative prognostic factors and adverse postoperative outcomes among older adults undergoing elective surgery. A recent narrative review on adverse postoperative outcomes among older adults included patients with different indications for surgery, such as hip fracture or other emergent procedures, and did not conduct meta-analyses of prognostic factors [75]. We targeted older adults undergoing elective surgery because of the potential to intervene to improve patient outcomes by identifying and optimizing these factors preoperatively. Multicomponent interventions aimed at improving a patient's nutrition, physical fitness, and cognition have shown promise in improving frailty [76]. Similarly, smoking status and depressive symptoms are potentially modifiable prognostic factors that were associated with developing postoperative complications. Interventions for preoperative smoking cessation have been associated with a lower risk of postoperative complications [77]. These prognostic factors could be targeted in the preoperative clinic.

There were limitations in our study's review process. Firstly, only studies that were published in English were included in this review to increase feasibility, but our findings are likely generalizable given the number of geographical regions represented in our systematic review. Secondly, there was substantial heterogeneity between studies for some outcomes, which could not always be adequately explored given a limited number of studies and a lack of individual patient-level data. Indeed, it is possible that by including such a broad spectrum of elective surgical procedures we may create difficulty in understanding exactly which prognostic factors are most likely to be important for certain patients, but this was explored in subgroup analyses and metaregression models, where possible. Additionally, this study was initiated prior to the introduction of the CHARMS checklist, which means that biases introduced in model development, validation, and evaluation of our included studies are less well described; however, we feel that we were able to identify important sources of selection bias, measurement bias, and confounding that threatened the validity of individual study findings [78].

There were also limitations imparted by the included studies themselves. The methodological quality assessment demonstrated that there were a number of studies reporting varying intensity of follow-up, which may have impacted the incidence of complications. The majority of studies included in this systematic review were cohort studies; therefore, our findings may be influenced by confounding. Sensitivity analyses demonstrated that our findings were largely consistent when only study-level effect estimates that were adjusted for potentially important confounders were included in the meta-analyses. Lastly, sometimes studies did not report independent variables for which there was a non-significant association with the dependent variable in the final multivariable model, which could potentially lead to a type 1 error in the findings of our meta-analyses. This is a limitation that is inherent in the prognosis literature that we 
hope will be overcome in the future by improved quality of reporting.

Our study had a number of strengths. There were 44 studies and over 12,000 patients included in our systematic review and meta-analyses, which allowed us to investigate a number of possible prognostic factors. The hypothesis-generating nature of this study allowed for the identification of prognostic factors that are potentially modifiable in the preoperative setting, which could lead to better surgical outcomes for older adults undergoing elective surgery.

\section{Conclusions}

In summary, this systematic review and meta-analysis highlights how common postoperative complications are among older adults undergoing elective surgery $(\mathrm{NNF}=4)$ and the importance of geriatric syndromes in identifying older adults at risk of harm. Furthermore, there were several prognostic factors identified that could be modifiable in a preoperative setting, including smoking and frailty, which can be explored in future knowledge translation strategies to develop interventions aimed at mitigating the risk faced by older adults undergoing elective surgery.

\section{Additional files}

\section{Additional file 1: PRISMA Checklist. (DOCX $30 \mathrm{~kb}$ )}

Additional file 2: Appendix 1. MEDLINE search strategy. Appendix 2. Order preference for combining data types in meta-analyses. Appendix $\mathbf{3}$. Data imputation methods. Appendix 4. Cochrane risk of bias assessment for randomized trials. Appendix 5. Newcastle-Ottawa scale for evaluating the quality of cohort studies. Appendix 6. Table of characteristics of prospective studies reporting prognostic factors associated with postoperative complications among older adults undergoing elective surgery. Appendix 7. Forest plots of study-level and pooled effect estimates for prognostic factors associated with postoperative complications among older adults undergoing elective surgery. Appendix 8. Forest plots of the study-level and pooled effect estimates of the prognostic factors associated with postoperative mortality among older adults undergoing elective surgery. Appendix 9. Forest plot of study-level and pooled effect estimates for prognostic factors associated with prolonged hospitalization among older adults undergoing elective surgery. Appendix 10. Forest plot of study-level and pooled effect estimates for prognostic factors associated with destination at discharge from hospital among older adults undergoing elective surgery (DOCX 550 kb)

\section{Abbreviations \\ ADL: activities of daily living; ASA: American Society of Anesthesiologists; Cl: confidence interval; ECOG: Eastern Cooperative Oncology Group; GDS: geriatric depression scale; HR: hazard ratio; IADL: instrumental activities of daily living; IQR: interquartile range; MMSE: Mini Mental State Exam; NNF: number needed to follow; OR: odds ratio; RCT: randomized controlled trial; RR: relative risk.}

\section{Acknowledgements}

We would like to thank Laure Perrier and Alissa Epworth for conducting the literature searches, Bianca Petrut and Lubna Al-Ansary for helping with article screening, and Susan Le for formatting of the manuscript.

\section{Funding}

JW is funded by the Canadian Institutes of Health Research Frederick Banting and Charles Best Canada Graduate Scholarship (Master's Award), and the Eliot Phillipson Clinician Scientist Training Program. ACT is funded by a Tier 2 Canada Research Chair in Knowledge Synthesis. SES is funded by a Tier 1 Canada Research Chair in Knowledge Translation.

\section{Availability of data and materials}

The full dataset is available from the corresponding author upon reasonable request.

\section{Role of the funder}

The funder had no role in the design and conduct of the study; collection, management, analysis, and interpretation of the data; preparation, review, or approval of the manuscript; or decision to submit the manuscript for publication.

\section{Authors' contributions}

JW, ACT, and SES designed the study. JW, ACT, CTH, PR, AG, CW, DS, and SES screened articles for inclusion. JW, ACT, CTH, PR, AG, CW, and DS abstracted data from included studies. JW and BP completed the data analysis. JW and SES drafted the manuscript. JW, ACT, CTH, BP, PR, AG, CW, DS, and SES edited the manuscript. All authors read and approved the final manuscript prior to its submission. All authors, external and internal, had full access to all of the data (including statistical reports and tables) in the study and can take responsibility for the integrity of the data and the accuracy of the data analysis.

Ethics approval and consent to participate Not required.

\section{Consent for publication}

Not applicable.

\section{Competing interests}

Dr. Andrea C. Tricco is a member of the editorial board of BMC Medicine; however, none of the other authors have any potential (or perceived) conflicts of interest.

\section{Publisher's Note}

Springer Nature remains neutral with regard to jurisdictional claims in published maps and institutional affiliations.

\section{Author details}

${ }^{1}$ Division of Geriatric Medicine, University of Toronto, 27 King's College Circle, Toronto, Ontario M5S 1A1, Canada. ${ }^{2}$ Institute for Health Policy, Management and Evaluation, University of Toronto, 4th Floor, 155 College Street, Toronto, Ontario M5T 3M6, Canada. ${ }^{3} \mathrm{Li}$ Ka Shing Knowledge Institute, St. Michael's Hospital, 209 Victoria Street, Toronto, Ontario M5B 1W8, Canada. ${ }^{4}$ Epidemiology Division, Dalla Lana School of Public Health, University of Toronto, Health Sciences Building, 155 College Street, 6th floor, Toronto, Ontario M5T 3M7, Canada. ${ }^{5}$ Toronto Health Economics and Technology Assessment Collaborative, Faculty of Pharmacy and Institute of Health Policy Management Evaluation, University of Toronto, 144 College Street, Toronto, Ontario M5S 3M2, Canada. ${ }^{6}$ Telfer School of Management, University of Ottawa, 55 Laurier Avenue East, Ottawa, Ontario K1N 6N5, Canada.

Received: 28 June 2017 Accepted: 5 December 2017

Published online: 12 January 2018

\section{References}

1. Audisio RA, Pope D, Gennari R, Maffezzini M, Hoekstra HJ, Mobarak D, et al. Shall we operate? Preoperative assessment in elderly cancer patients (PACE) can help. A SIOG surgical task force prospective study. Crit Rev Oncol Hematol. 2008;65:156-63.

2. Badgwell B, Stanley J, Chang GJ, Katz MHG, Lin HY, Ning J, et al. Comprehensive geriatric assessment of risk factors associated with adverse outcomes and resource utilization in cancer patients undergoing abdominal surgery. J Surg Oncol. 2013;108:182-6.

3. Sundermann SH, Dademasch A, Seifert B, Biefer HRC, Emmert MY, Walther $\mathrm{T}$, et al. Frailty is a predictor of short- and mid-term mortality 
after elective cardiac surgery independently of age. Interact Cardiovasc Thorac Surg. 2014;18:580-5.

4. Bakker RC, Osse RJ, Tulen JHM, Kappetein AP, Bogers AJJC. Preoperative and operative predictors of delirium after cardiac surgery in elderly patients. Eur J Cardiothorac Surg. 2012;41:544-9.

5. Veliz-Reissmuller GT, Aguero Torres $H$, van der Linden J, Lindblom D, Eriksdotter Jonhagen M. Pre-operative mild cognitive dysfunction predicts risk for post-operative delirium after elective cardiac surgery. Aging Clin Exp Res. 2007:19:172-7.

6. Kristjansson SR, Jordhøy MS, Nesbakken A, Skovlund E, Bakka A, Johannessen $\mathrm{H}-\mathrm{O}$, et al. Which elements of a comprehensive geriatric assessment (CGA) predict post-operative complications and early mortality after colorectal cancer surgery? J Geriatr Oncol. 2010;1 (2):57-65.

7. Dasgupta M, Rolfson DB, Stolee P, Borrie MJ, Speechley M. Frailty is associated with postoperative complications in older adults with medical problems. Arch Gerontol Geriatr. 2009;48:78-83.

8. Marcantonio ER, Goldman L, Mangione CM, Ludwig LE, Muraca B, Haslauer $C M$, et al. A clinical prediction rule for delirium after elective noncardiac surgery. JAMA. 1994;271(2):134-9.

9. Mohanty S, Rosenthal RA, Russell MM, Neuman MD, Ko CY, Esnaola NF. Optimal perioperative management of the geriatric patient: a best practices guideline from the American College of Surgeons NSQIP and the American Geriatrics Society. J Am Coll Surg. 2016;222(5):930-47.

10. Liberati A, Altman DG, Tetzlaff J, Mulrow C, Gotzsche PC, loannidis JP, et al. The PRISMA statement for reporting systematic reviews and meta-analyses of studies that evaluate health care interventions: explanation and elaboration. J Clin Epidemiol. 2009;62(10):e1-34.

11. Stroup DF, Berlin JA, Morton SC, Olkin I, Williamson GD, Rennie D, et al. Meta-analysis of observational studies in epidemiology: a proposal for reporting. Meta-analysis Of Observational Studies in Epidemiology (MOOSE) group. JAMA. 2000;283(15):2008-12

12. World Health Organization. Definition of an Older or Elderly Person. Geneva: WHO 2010. http:/www.who.int/healthinfo/survey/ageingdefnolder/en/index.html. Accessed 21 Dec 2017.

13. United Nations, Department of Economic and Social Affairs, Population Division. World Population Ageing 2013. ST/ESA/SER.A/348. 2013. http:// www.un.org/en/development/desa/population/publications/ageing/ WorldPopulationAgeingReport2013.shtml. Accessed 21 Dec 2017.

14. Marcantonio ER, Flacker JM, Wright RJ, Resnick NM. Reducing delirium after hip fracture: a randomized trial. J Am Geriatr Soc. 2001;49(5):516-22.

15. Lenartowicz M, Parkovnick M, McFarlan A, Haas B, Straus SE, Nathens AB, et al. An evaluation of a proactive geriatric trauma consultation service. Ann Surg. 2012;256(6):1098-101.

16. Wells G, Shea B, O'Connell D, Peterson J, Welch V, Losos M, et al. The NewcastleOttawa Scale (NOS) for Assessing the Quality of Nonrandomised Studies in Meta-Analyses. Ottawa, Ontario: Ottawa Hospital Research Institute; 2008.

17. Higgins JP, Altman DG, Gotzsche PC, Juni P, Moher D, Oxman AD, et al. The Cochrane Collaboration's tool for assessing risk of bias in randomised trials. BMJ. 2011;343:d5928.

18. EPOC Risk of Bias Tool. Cochrane Effective Practice and Organization Care Group. 2011. http://epoc.cochrane.org/sites/epoc.cochrane.org/files/ uploads/Risk\%20of\%20Bias\%2005-01-2009.doc.

19. Sanchez-Meca J, Marin-Martinez F, Chacon-Moscoso S. Effect-size indices for dichotomized outcomes in meta-analysis. Psychol Methods. 2003;8(4):448-67.

20. Mayer D. Essential Evidence-Based Medicine. 2nd ed. Cambridge: Cambridge University Press; 2009.

21. Cochrane Handbook for Systematic Reviews of Interventions Version 5.1.0 [updated March 2011]. The Cochrane Collaboration, 2011. www. handbook.cochrane.org. Accessed 21 Dec 2017.

22. Viechtbauer W. Conducting meta-analyses in $\mathrm{R}$ with the metafor package. J Stat Softw. 2010;36(3):48

23. Schwarzer G. meta: An R package for meta-analysis. R News. 2007;7(3):40-5.

24. Clement ND, MacDonald D, Howie CR, Biant LC. The outcome of primary total hip and knee arthroplasty in patients aged 80 years or more. J Bone Joint Surg Br. 2011;93(9):1265-70.

25. Robinson TN, Wu DS, Pointer LF, Dunn CL, Moss M. Preoperative cognitive dysfunction is related to adverse postoperative outcomes in the elderly. J Am Coll Surg. 2012;215(1):12-7.

26. Fukuse T, Satoda N, Hijiya K, Fujinaga T. Importance of a comprehensive geriatric assessment in prediction of complications following thoracic surgery in elderly patients. Chest. 2005;127:886-91.
27. Papaioannou A, Fraidakis O, Michaloudis D, Balalis C, Askitopoulou H. The impact of the type of anaesthesia on cognitive status and. Eur J Anaesthesiol. 2005;22:492-9.

28. Suh DH, Kim JW, Kim HS, Chung HH, Park NH, Song YS. Pre- and intraoperative variables associated with surgical complications in elderly patients with gynecologic cancer: the clinical value of comprehensive geriatric assessment. J Geriatr Oncol. 2014;5(3):315-22.

29. Makary MA, Segev DL, Pronovost PJ, Syin D, Bandeen-Roche K, Patel P, et al. Frailty as a predictor of surgical outcomes in older patients. J Am Coll Surg. 2010;210(6):901-8.

30. Gerude MF, Dias FL, de Farias TP, Albuquerque Sousa B, Thuler LC Predictors of postoperative complications, prolonged length of hospital stay, and short-term mortality in elderly patients with malignant head and neck neoplasm. ORL J Otorhinolaryngol Relat Spec. 2014;76(3):153-64.

31. Huisman MG, Van Leeuwen BL, Ugolini G, Montroni I, Spiliotis J, Stabilini C, et al. Timed Up \& Go': A screening tool for predicting 30-day morbidity in onco-geriatric surgical patients? A multicenter cohort study. PLoS One. 2014;9(1):e86863.

32. Javierre C, Ricart A, Manez R, Farrero E, Carrio ML, Rodriquez-Castro D, et al. Age and sex differences in perioperative myocardial infarction after cardiac surgery. Interact Cardiovasc Thorac Surg. 2012;15(1):28-32.

33. Zhang L, Wang C, Sha SY, Kwauk S, Miller AR, Xie MS, et al. Mini-nutrition assessment, malnutrition, and postoperative complications in elderly Chinese patients with lung cancer. J BUON. 2012;17(2):323-6.

34. Aykut K, Albayrak G, Guzeloglu M, Baysak A, Hazan E. Preoperative mild cognitive dysfunction predicts pulmonary complications after coronary artery bypass graft surgery. J Cardiothorac Vasc Anesth. 2013;27(6):1267-70.

35. Lasithiotakis K, Petrakis J, Venianaki M, Georgiades G, Koutsomanolis D, Andreou A, et al. Frailty predicts outcome of elective laparoscopic cholecystectomy in geriatric patients. Surg Endosc. 2013;27(4):1144-50.

36. Kenig J, Olszewska U, Zychiewicz B, Barczynski M, Mitus-Kenig M. Cumulative deficit model of geriatric assessment to predict the postoperative outcomes of older patients with solid abdominal cancer. J Geriatr Oncol. 2015;6:370-9.

37. Kim S, Marsh AP, Rustowicz L, Roach C, Leng XI, Kritchevsky SB, et al. Selfreported mobility in older patients predicts early postoperative outcomes after elective noncardiac surgery. Anesthesiology. 2016;124:815-25.

38. Courtney-Brooks M, Tellawi AR, Scalici J, Duska LR, Jazaeri AA, Modesitt SC, et al. Frailty: an outcome predictor for elderly gynecologic oncology patients. Gynecol Oncol. 2012;126:20-4.

39. Green P, Woglom AE, Genereux P, Daneault B, Paradis JM, Schnell S, et al. The impact of frailty status on survival after transcatheter aortic valve replacement in older adults with severe aortic stenosis: a single-center experience. JACC Cardiovasc Interv. 2012;5:974-81.

40. Kim S-W, Han H-S, Jung H-W, Kim K-I, Hwang DW, Kang S-B, et al. Multidimensional frailty score for the prediction of postoperative mortality risk. JAMA Surg. 2014;149:633-40.

41. Tan KY, Kawamura YJ, Tokomitsu A, Tang T. Assessment for frailty is useful for predicting morbidity in elderly patients undergoing colorectal cancer resection whose comorbidities are already optimized. Am J Surg. 2012;204:139-43.

42. Goto T, Baba T, Ito A, Maekawa K, Koshiji T. Gender differences in stroke risk among the elderly after coronary artery surgery. Anesth Analg. 2007;104(5):1016-22.

43. Dales RE, Dionne G, Leech JA, Lunau M, Schweitzer I. Preoperative prediction of pulmonary complications following thoracic surgery. Chest. 1993;104(1):155-9.

44. Gerson MC, Hurst JM, Hertzberg VS, Doogan PA, Cochran MB, Lim SP, et al. Cardiac prognosis in noncardiac geriatric surgery. Ann Intern Med. 1985; 103(6 (Pt 1)):832-7.

45. Kothari A, Phillips S, Bretl T, Block K, Weigel T. Components of geriatric assessments predict thoracic surgery outcomes. J Surg Res. 2011;166(1):5-13.

46. van Venrooij LM, van Leeuwen PA, de Vos R, Borgmeijer-Hoelen MM, de Mol BA. Preoperative protein and energy intake and postoperative complications in well-nourished, non-hospitalized elderly cardiac surgery patients. Clin Nutr. 2009;28(2):117-21.

47. Fried LP, Tange CM, Walston J, Newman AB, Hirsch C, Gottdiener J, et al. Frailty in older adults: evidence for a phenotype. J Gerontol A Biol Sci Med Sci. 2001;56A(3):M146-56.

48. Rolfson DB, Majumdar SR, Tsuyuki RT, Tahir A, Rockwood K. Validity and reliability of the Edmonton Frail Scale. Age Ageing. 2006;35(5):526-9. 
49. Yesavage JA, Brink TL, Rose TL, Lum O, Huang V, Adey M, et al. Development and validation of a geriatric depression screening scale: a preliminary report. J Psychiatr Res. 1982;17(1):37-49.

50. Goldman L, Caldera DL, Nussbaum SR, Southwick FS, Krogstad D, Murray B, et al. Multifactorial index of cardiac risk in noncardiac surgical procedures. $N$ Engl J Med. 1977;297(16):845-50.

51. Kim K-I, Park K-H, Koo K-H, Han H-S, Kim C-H. Comprehensive geriatric assessment can predict postoperative morbidity and mortality in elderly patients undergoing elective surgery. Arch Gerontol Geriatr. 2013;56:507-12.

52. Hattler BG, Madia C, Johnson C, Armitage JM, Hardesty RL, Kormos RL, et al. Risk stratification using the Society of Thoracic Surgeons Program. Ann Thorac Surg. 1994;58(5):1348-52.

53. Williams JB, Alexander KP, Morin JF, Langlois $Y$, Noiseux N, Perrault LP, et al. Preoperative anxiety as a predictor of mortality and major morbidity in patients aged $>70$ years undergoing cardiac surgery. Am J Cardiol. 2013;111(1):137-42.

54. Posner BM, Jette AM, Smith KW, Miller DR. Nutrition and health risks in the elderly: the nutrition screening initiative. Am J Public Health. 1993;83(7):972-8.

55. Tamburino C, Capodanno D, Ramondo A, Petronio AS, Ettori F, Santoro G, et al. Incidence and predictors of early and late mortality after transcatheter aortic valve implantation in 663 patients with severe aortic stenosis. Circulation. 2011;123(3):299-308.

56. Wenaweser P, Pilgrim T, Roth N, Kadner A, Stortecky S, Kalesan B, et al: Clinical outcome and predictors for adverse events after transcatheter aortic valve implantation with the use of different devices and access routes. Am Heart J. 2011;161(6):1114-24.

57. Betomvuko P, Michaux I, Gabriel L, Bihin B, Gourdin M, De Saint Hubert M. Gait speed as predictor of outcomes of elective cardiac surgery in older patients. Eur Geriatric Med. 2015;6(1):S147.

58. Rogers MPL, Matthew $\mathrm{H}$, Daltroy Lawren $\mathrm{H}$, Eaton $\mathrm{H}$, Peteet J, Wright $\mathrm{E}$, Albert M. Delirium after elective orthopedic surgery: risk factors and natural history. Int J Psychiatry Med. 1989;19:109-21.

59. Reinöhl J, Kaier K, Gutmann A, Sorg S, von Zur MC, Siepe M, et al. Inhospital resource utilization in surgical and transcatheter aortic valve replacement. BMC Cardiovasc Disord. 2015;15:132.

60. Schmidt M, Eckardt R, Scholtz K, Neuner B, Von Dossow-Hanfstingl V, Sehouli J, et al. Patient empowerment improved Perioperative quality of care in cancer patients aged $>65$ Years - a randomized controlled trial. PLoS One. 2015;10(9):e0137824.

61. Blakoe $\mathrm{M}$, Greve $\mathrm{H}$. Frailty - preoperative assessment and implications for nursing practice following heart surgery. Eur J Cardiovasc Nursing. 2015;14:S44.

62. Kosar CM, Tabloski PA, Travison TG, Jones RN, Schmitt EM, Puelle MR, et al. Effect of preoperative pain and depressive symptoms on the risk of postoperative delirium: A prospective cohort study. Lancet Psychiatry. 2014;1:431-6.

63. Lawrence VA, Hazuda HP, Cornell JE, Pederson T, Bradshaw PT, Mulrow CD, et al. Functional independence after major abdominal surgery in the elderly. J Am Coll Surg. 2004:199:762-72.

64. Amemiya $T$, Oda $K$, Ando M, Kitagawa $Y$, Shimada H, Kuroiwa K, et al. Activities of daily living and quality of life of elderly patients after elective surgery for gastric and colorectal cancers. Ann Surg. 2007;246:222-8.

65. Min L, Mazzurco L, Gure TR, Cigolle CT, Lee P, Bloem C, et al. Longitudinal functional recovery after geriatric cardiac surgery. J Surg Res. 2015;194(1):25-33.

66. Legner VJ, Doerner D, Reilly DF, Mccormick WC. Risk factors for nursing home placement following major nonemergent surgery. Am J Med. 2004;117:82-6.

67. Otomo S, Maekawa K, Goto T, Baba T, Yoshitake A. Pre-existing cerebral infarcts as a risk factor for delirium after coronary artery bypass graft surgery. Interact Cardiovasc Thorac Surg. 2013;17(5):799-804.

68. Pirracchio R, Resche-Rigon M, Bresson D, Basta B, Welschbillig S, Heyer L, et al. One-year outcome after neurosurgery for intracranial tumor in elderly patients. J Neurosurg Anesthesiol. 2010;22(4):342-6.

69. Hoogerduijn JG, de Rooij SE, Grobbee DE, Schuurmans MJ. Predicting functional decline in older patients undergoing cardiac surgery. Age Ageing. 2014;43(2):218-21.

70. Kwon S, Symons R, Yukawa M, Dasher N, Legner V, Flum DR. Evaluating the association of preoperative functional status and postoperative functional decline in older patients undergoing major surgery. Am Surg. 2012;78(12):1336-44.

71. Fitz-Henry J. The ASA, classification and peri-operative risk. Ann R Coll Surg Engl. 2011;93:185-7.

72. Clegg A, Young J, lliffe S, Rikkert MO, Rockwood K. Frailty in elderly people. Lancet. 2013;381:752-62.
73. Fried LP, Tange CM, Walston J, Newman AB, Hirsch C, Gottdiener J, et al. Frailty in older adults: evidence for a phenotype. J Gerontol Med Sci. 2001;56A(3):M146-56.

74. Rockwood K, Song X, MacKnight C, Bergman H, Hogan DB, McDowell I, et al. A global clinical measure of fitness and frailty in elderly people. CMAJ. 2005;173(5):489-95.

75. Oresanya LB, Lyons WL, Finlayson E. Preoperative assessment of the older patient: a narrative review. JAMA. 2014;311:2110-20.

76. Ng T, Feng L, Nyunt M, Feng L, Niti M, Tan B, et al. Nutritional, physical, cognitive, and combination interventions and frailty reversal among older adults: a randomized controlled trial. Am J Med. 2015;128(11):1225-36.

77. Thomsen T, Villebro N, Moller AM. Interventions for preoperative smoking cessation. Cochrane Database Syst Rev. 2014;3:CD002294.

78. Moons KGM, Groot JAH, Bouwmeester W, Vergouwe Y, Mallett S, Altman DG et al. Critical appraisal and data extraction for systematic reviews of prediction modelling studies: the CHARMS checklist. PLoS Med. 2014;11(10):e1001744.

\section{Submit your next manuscript to BioMed Central and we will help you at every step:}

- We accept pre-submission inquiries

- Our selector tool helps you to find the most relevant journal

- We provide round the clock customer support

- Convenient online submission

- Thorough peer review

- Inclusion in PubMed and all major indexing services

- Maximum visibility for your research

Submit your manuscript at www.biomedcentral.com/submit
) Biomed Central 